DE91 000479

\title{
SWING-FREE MOVEMENT OF SIMPLY SUSPENDED OBJECTS EMPLOYING PARAMETER ESTIMATION
}

\author{
Jill C. Werner \\ Rush D. Robinett \\ Ben J. Petterson \\ Sandia National Laboratories \\ Albuquerque, NM 87185
}

\begin{abstract}
An adaptive, swing-free trajectory planner for a gantry robot has been analytically developed and experimentally implemented on a commercial robot. A batch, nonlinear least square estimator (differential correction) is used to predict the initial conditions, mass, and frequency of the simply suspended object from a set of force sensor measurements. These parameters, in turn, are supplied to the swing-free trajectory planner to perform the maneuver. These algorithms have been implemented on a CIMCORP XR6100' gantry robot, and swing-free trajectories have been performed by the robot using the adaptive trajectory planner.
\end{abstract}

IXR is a registered trademark of CIMCORP Inc. 


\section{Contents}

1 Introduction 5

2 Mathematical Foundation 5

3 Experimental Implementation : 7

3.1 Hardware ........... 7

3.2 Software ............. 8

4 Results 8

5 Conclusions 9

\section{List of Figures}

1 Diagram of Transporting Device . 5

2. Gantry Robot with Force Sensor . 6

3 Coordinate System and Force Diagram ........... 6

4 Suspended Objects Used in Experiments ............ 8

\section{List of Tables}

1 Measured and Calculated Periods of Test Ohjects ........... 


\section{Introduction}

Sandia National Laboratories is currently investigating the feasibility of using intelligent machines at nuclear waste storage sites. One class of operations that is necessary at a waste storage site is the transportation of nuclear waste shipping casks and other heavy objects to various locations throughout the installation. During overhead crane transportation, an object is free to swing. If any oscillation of the object begins, it must be sufficiently damped by the operator, or allowed to decay naturally before the next operation can begin. Either option is time consuming and reduces the availability of the facility. However, damping the oscillation of simply suspended payloads during transportation is possible if the acceleration of the crane is programmable. Jones and Petterson [1] discuss acceleration profile shaping, for a trajectory planner in detail and define the necessary conditions for swing-free transportation.

This paper presents an extension of their work: the analytical development and experimental implementation of an adaptive, swing-free trajectory planner. Other researchers, for example Tzes and Yurkovich [2] and Nelson and Mitra [3], have developed parameter estimation schemes for closed-loop control of flexible structures. Our open-loop planner is made adaptive by employing a batch, nonlinear least square estimator to predict the parameters of the suspended object which are necessary for swing-free motion from a set of force sensor measurements. Initially, an object with unknown parameters is picked up and moved (which causes the object to oscillate) and force measurements are taken. Next, the estimator processes the data and extracts the parameters of interest while the force-damping (closedloop) controller [4] damps the object's oscillation. Finally, the planner calculates the swing-free trajectory from the estimated parameters and instructs the robot to move. Note that the only required parameter for swing-free motion is the period of oscillation [1], but the estimator includes the initial conditions and the mass of the suspended object to increase the robustness of the

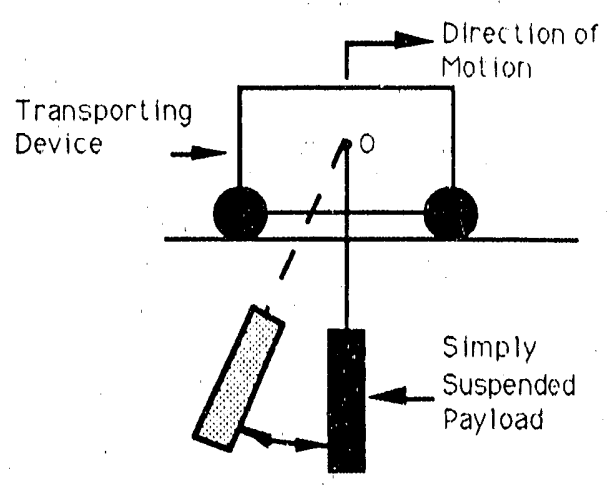

Figure 1: Diagram of Transporting Device

estimation process. The robustiness of the estimator became an issue when a simple scheme to calculate the period of the pendulum (measure the time between peaks of the force sensor out. put) did not produce adequate results. 'The simple scheme was limited in resolution by the $47 \mathrm{nns}$ update rate of data sampling and subject to $47 \mathrm{~ms}$ increments of error due to noise in the data.

\section{Mathematical Foundation}

There are many ways to produce a swing-free move of a simply suspended object (Figure 1). One of the most general methods is described by Jones and Petterson [1] where a double pulse train is employed. The basic ingredient necessary for every approach to developing swing-free trajectories is knowledge of the frequency of oscillation of the suspended object. Consequently, the period of oscillation must be measured each time a different object is moved in order to produce a swing-free move. In this section, wo describe a batch, nonlinear least square estimator that enables the computer to automatically cal. culate the period of oscillation of the suspended object from force sensor measurements and pro. duce swing-free trajectories.

The nonlinear least square estimator is bascd on the Gaussian least square differontial correction (GLSDC) algorithm described by Junkins [5]. The GLSDC algorithm is chosen because it is easy to implement and highly flr xible (refer to Reverences [6] and [7]). The algorithm applies 
a generalization of Newton's root solving method to obtain the nonlinear solution from a succession of locally linearized least squares solutions. In other words, the nonlinear measurement model (in terms of the estimation parameters),

$$
\underline{Y}=E(\underline{X})
$$

where

$\underline{Y}$ - computed measurement vector $(p \times 1)$

F - nonlinear transformation vector $(p \times 1)$

$\underline{X}$ - estimation parameter vector $(n \times 1)$,

is linearized about some nominal value, $\underline{x}_{0}$, to produce the Jacobian matrix, A,

$$
A=\left.\left[\frac{\partial \underline{Y}}{\partial \underline{\underline{X}}}\right]\right|_{\underline{X}_{0}}=\left.\left[\frac{\partial \underline{F}}{\partial \underline{\underline{X}}}\right]\right|_{\underline{X}_{0}}
$$

which is employed in the successive linear least squares estimate of $\underline{X}$ as

$$
\begin{gathered}
\underline{X}=\underline{X_{0}}+\Delta \underline{X} \\
\Delta \underline{X}=\left(A^{T} W^{T} A\right)^{-1} A^{T} W \Delta \underline{Y} \\
\Delta \underline{Y}=\underline{Y}-\underline{Y}
\end{gathered}
$$

where

$W$ - least squares weighting matrix, and

$\underline{\underline{Y}}$ - actual measurements.

Equation (4) is known as the normal equations (derived in Reference [5]), and $\tilde{Y}$ is a time sequence of measurements taken over a prescribed time interval at a given sampling rate.

To illustrate this mathematical development, the estimator formulation is applied to a force sensor located on the arm of the gantry robot (Figure 2) which provides the measurement data to estimate the mass, frequency, and two initial conditions of a planar pendulum. The actual measurements are $p$ vertical force $\left(F_{x}\right.$, Figure 3$)$ measurements, and the measurement model for these $p$ data points is given by

$$
\begin{gathered}
T=m g \cos \theta+m l \dot{\theta}^{2} \\
F_{x}=T \cos \theta=m g \cos ^{2} \theta+m l \dot{\theta}^{2} \cos \theta
\end{gathered}
$$

and

$$
\theta=\theta_{0} \cos \omega t+\frac{\dot{\theta}_{0}}{\omega} \sin \omega t
$$

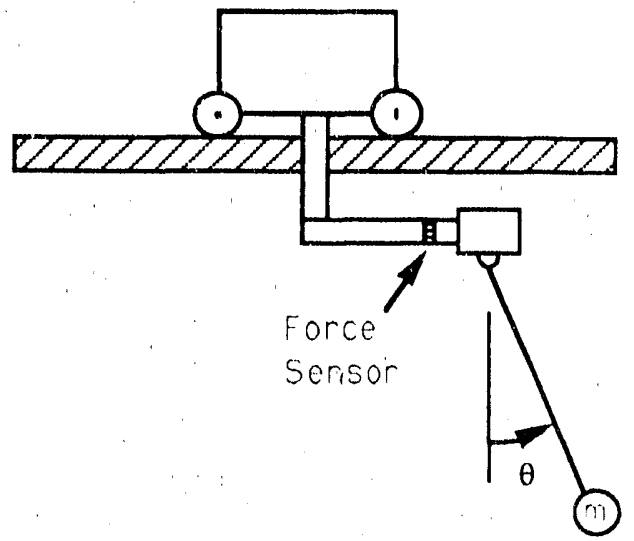

Figure 2: Gantry Robot with Force Sensor

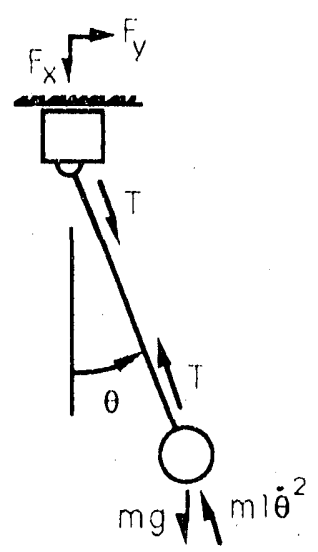

Figure 3: Coordinate System and Force Diagrall 
where

$m$ - mass of pendulum,

$l$ - length of pendulum

$\theta_{0}, \dot{\theta}_{0}$ - initial conditions of pendulum,

$g$ - gravity,

$T$ - cable tension, and

$\omega \quad$ - frequency $=\sqrt{g / l}$,

which produces the nonlinear measurement model

$$
Y\left(t_{i}\right)=F_{x}\left(t_{i}\right)=m g\left[\cos ^{2} \theta_{i}+\frac{\dot{\theta}_{i}^{2}}{\omega^{2}} \cos \theta_{i}\right],
$$

where $\theta_{i}=\theta_{0} \cos \omega t_{i}+\frac{\dot{\theta}_{0}}{\omega} \sin \omega t_{i}$.

Note, using only one channel of force data, $F_{x}$, simplifies the development of the estimator algorithm. While $F_{y}$ and $F_{z}$ forces are also available from our force sensor, our algorithm may be applied on equipment where the force from only one direction is available.

The weight matrix, $W$ in $\mathrm{Eq}(4)$, is a diagonal, positive definite, constant matrix, and the Jacobian matrix is analytically developed from $\mathrm{Eq}(9)$ by taking the first partial derivatives with respect to $\theta_{0}, \dot{\theta_{0}}, \mathrm{~m}$, and $\omega$.

$$
\begin{aligned}
& A=\left[\frac{\partial \underline{Y}}{\partial \theta_{0}} \frac{\partial \underline{Y}}{\partial \theta_{0}} \frac{\partial \underline{Y}}{\partial m} \frac{\partial \underline{Y}}{\partial \omega}\right] \\
& \frac{\partial \underline{Y}\left(t_{i}\right)}{\partial \theta_{o}}=\frac{\partial \underline{Y}}{\partial \theta_{i}} \frac{\partial \theta_{i}}{\partial \theta_{o}}+\frac{\partial \underline{Y}}{\partial \dot{\theta}_{i}} \frac{\partial \dot{\theta}_{i}}{\partial \theta_{o}}= \\
& m\left[-g \sin 2 \theta_{i} \cos \omega t_{i}\right. \\
& +l \cos \theta_{i}\left(2 \omega^{2} \theta_{0} \sin ^{2} \omega t_{i}-\omega \dot{\theta}_{0} \sin 2 \omega t_{i}\right) \\
& \left.-l \theta_{i} \cos \omega t_{i} \sin \theta_{i}\right] \\
& \frac{\partial \underline{Y}\left(t_{i}\right)}{\partial \dot{\theta}_{o}}=\frac{\partial \underline{Y}}{\partial \theta_{i}} \frac{\partial \theta_{i}}{\partial \dot{\theta}_{0}}+\frac{\partial \underline{Y}}{\partial \dot{\theta}_{i}} \frac{\partial \dot{\theta}_{i}}{\partial \dot{\theta}_{o}}= \\
& m\left(-\frac{g}{\omega} \sin 2 \theta_{i} \sin \omega t_{i}\right. \\
& +l \cos \theta_{i}\left[-\omega \theta_{0} \sin 2 \omega t_{i}+2 \dot{\theta_{0}} \cos ^{2} \omega t_{i}\right] \\
& \left.-\frac{l}{\omega} \dot{\theta}_{i}^{2} \sin \theta_{i} \sin \omega t_{i}\right) \\
& \frac{\partial \underline{Y}\left(t_{i}\right)}{\partial m}=\frac{\partial \underline{Y}}{\partial \theta_{i}} \frac{\partial \theta_{i}}{\partial m}+\frac{\partial \underline{Y}}{\partial \dot{\theta}_{i}} \frac{\partial \dot{\theta}_{i}}{\partial m}= \\
& g \cos ^{2} \theta_{i}+l\left(\dot{\theta_{i}^{2}}\right) \cos \theta_{i}
\end{aligned}
$$

$$
\begin{aligned}
& \frac{\partial \underline{Y}\left(t_{i}\right)}{\partial \omega}=\frac{\partial \underline{Y}}{\partial \theta_{i}} \frac{\partial \theta_{i}}{\partial \omega}+\frac{\partial \underline{Y}}{\partial \dot{\theta}} \frac{\partial \dot{\theta}_{i}}{\partial \omega}= \\
& m g\left[-\sin 2 \theta_{i}\left(-t_{i} \theta_{o} \sin \omega t_{i}-\frac{\dot{\theta}_{o}}{\omega^{2}} \sin \omega t_{i}\right.\right. \\
& \left.+\frac{\dot{\theta}_{o}}{\omega} t_{i} \cos \omega t_{i}\right)+\cos \theta_{i}\left(-\frac{2 \theta_{o} \dot{\theta}_{o}}{\omega} t_{i} \cos 2 \omega t_{i}\right. \\
& \left.\left(\theta_{o}^{2} t_{i}+\frac{\theta_{o} \dot{\theta}_{o}}{\omega^{2}}-\frac{\dot{\theta}_{o}^{2}}{\omega^{2}} t_{i}\right) \sin 2 \omega t_{i}-\frac{2 \dot{\theta}_{o}^{2}}{\omega^{3}} \cos ^{2} \omega t_{i}\right) \\
& -\frac{\dot{\theta}_{i}^{2}}{\omega^{2}} \sin \theta_{i}\left(-t_{i} \theta_{o} \sin \omega t_{i}-\frac{\dot{\theta}_{o}}{\omega^{2}} \sin \omega t_{i}\right. \\
& \left.\left.+\frac{\dot{\theta}_{o}}{\omega} t_{i} \cos \omega t_{i}\right)\right]
\end{aligned}
$$

Equations (9) and (10), the weight matrix, and the force sensor measurements are the required elements of the GLSDC algorithm that provides the estimate of the frequency (period of oscillation) to the swing-iree trajectory planner. The computer implementation of the GLSDC algorithm is discussed in the next section.

\section{Experimental Implementation}

\subsection{Hardware}

The adaptive, swing-free trajectory planner is implemented on a CIMCORP XR6100 robot at Sandia National Laboratories. A simply suspended object is held hy the robot gripper, and a force-moment sensor (manufactured by JR3 Corporation) capable of transducing the forces in the three principal axes and the corresponding moments is used to monitor the oscillations of the payload. Four different suspended objects are used to test the adaptive, swing-free planner: a $22.7 \mathrm{~kg}$ ( 50 pound) weight suspended from a $1.93 \mathrm{~m}$ ( 76 inch) cable, a $45.4 \mathrm{~kg}$ (100 pound) weight suspended first from a $1.83 \mathrm{~m}$ (72 inch) cable and then from a $0.74 \mathrm{~m}$ ( $29 \mathrm{inch}$ ) cable, and a $1 / 5$ scale ( $1 \mathrm{~m}$ ( 39 inches) tall) $11.4 \mathrm{~kg}$ ( 25 pound) model of a fuel rod assembly on a pivot joint ( $\mathrm{sec}$ Figure 4). The readings from the force sensor are 


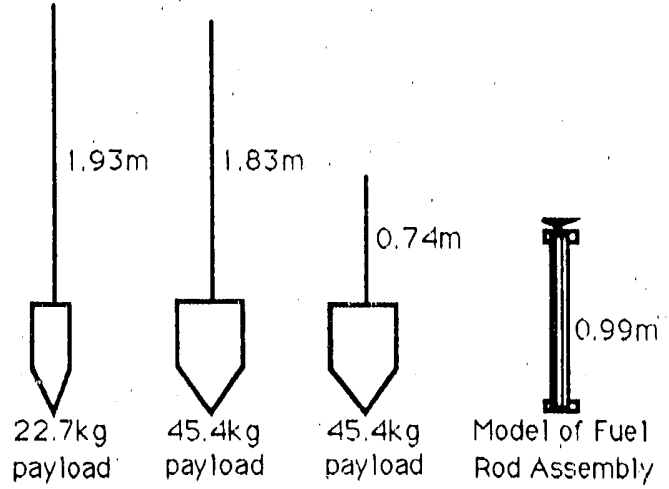

Figure 4: Suspended Objects Used in Experiments

stored on a Digital Equipment Corporation LSI$11 / 73$ executing the $\mathrm{R} T-11^{2}$ real time operating system which is also used to implement swingfree moves of the suspended objects. Finally, the batch estimation program is run on a $\mathrm{SUN}-3^{3}$ computer that is networked with the LSI-11.

\subsection{Software}

The first step in executing the adaptive, swingfree trajectory planner is to gather force data while the pendulum is swinging. The suspended object is picked up, displaced, and allowed to swing freely. A set of 60 force readings (sufficient to assure a full period of oscillation for any of our payloads) is taken as the payload swings with each reading at an interval of 4 ? milliseconds which is the update rate of the robot controller. Synchronizing our readings with the controller updates allows us to be certain of the length of time between readings. Once a set of force data has been recorded, a force servo damping program, which uses a technique described by Jones, Petterson, and Werner [2], is used to damp out the swinging motion of the suspended object.

The batch estimator runs while the robot damps the payload's oscillation. The constants necessary for the computations are a convergence criteria, the weighting matrix $(W)$, gravity, a

\footnotetext{
${ }^{2}$ LSI-11/73 and RT-11 are registered trademarks of Digital Equipment Corporation.

${ }^{3}$ SUN-3 is a registered trademark of Sun Microsystems.
}

maximum number of iterations, the number of force readings $(p)$, and the sampling period. The values needed to initialize the program variables are the initial guesses of the calculated parameters and a set of force readings $(\underline{\underline{r}})$. The calculated parameters $(\underline{X})$ are the initial angle and initial angular velocity of the suspended object, its mass, and its frequency. In each iteration, the batch estimator calculates the matrices $A^{T} W A$ and $A^{T} W \Delta \underline{Y}$ of $\mathrm{Eq}$ (4) (using Eqs (11 - 14) to calculate the Jacobian matrix, $A$ ), solves this linear system of equations using $L U$ decomposition [8] for the change in the desired parameters $(\Delta \underline{X})$, and adjusts the values of the desired parameters by adding in this change. The iteration: conclude when there is negligible change in the values of the desired parameters (i.e., $\Delta \underline{X} \equiv 0$ ), or when the performance function gets smaller than the convergence criteria. The object's frequency is then converted into its period and passed into the swing-free trajectory program on the LSI-11.

The trajectory is computed using the acceieration profile tailoring described by Jones and Petterson [1], and is sent to the robot controller as a series of displacement commands. The communication between the robot controller and the LSI-11 control computer is synchronized by the robot controller through the Realtime Path Modification (RPM) board. The RPM board sends a synchronizing pulse to the control computer every 47 milliseconds. When the swing-free trajectory program senses the synchronization pulse, it transmits a position update over a serial communication line to the RPM board, which then commands the robot to move. This process continues until the desired trajectory is complete.

\section{Results}

Tests of the adaptive, swing-free trajectory planner were done using the four different suspended objects. Each object's period was measured by timing its oscillations, and then calculated using the estimator. A comparison of these two periods and the resulting residual oscillations after a swing-free move can be seen in Table 1. As shown in the table, the calculated periods are within two 


\begin{tabular}{|l|c|c|c|c|c|}
\hline Test Object & $\begin{array}{c}\text { Length } \\
(\mathrm{m})\end{array}$ & $\begin{array}{c}\text { Measured } \\
\text { Period } \\
(\mathrm{sec})\end{array}$ & $\begin{array}{c}\text { Average } \\
\text { Calculated } \\
\text { Period } \\
(\mathrm{sec})\end{array}$ & $\begin{array}{c}\text { Residual } \\
\text { Oscillation } \\
\text { w/measured } \\
\text { (degrees) }\end{array}$ & $\begin{array}{c}\text { Residual } \\
\text { Oscillation } \\
\text { w/calculated } \\
\text { (degrees) }\end{array}$ \\
\hline $22.7 \mathrm{~kg}$ payload & 1.93 & 2.79 & 2.80 & 0.05 & 0.09 \\
$45.4 \mathrm{~kg}$ payload & 1.83 & 2.71 & 2.77 & 0.05 & 0.10 \\
$45.4 \mathrm{~kg}$ payload & 0.74 & 1.75 & 1.76 & 0.15 & 0.31 \\
$11.4 \mathrm{~kg}$ Fuel rod & 1.0 & 1.64 & 1.66 & 0.14 & 0.27 \\
assembly model & & - & - & 6.8 & - \\
\hline 45.4kg payload, no & & -93 & - & & \\
oscillation damping & 1.93 & & \\
\hline
\end{tabular}

Table 1: Measured and Calculated Periods of Test Objects

percent of the measured values, and although the resulting residual oscillations for the calculated periods are about double the value of those with the measured periods, both are over $95 \%$ smaller than the oscillation that occurs with an uncontrolled move (last entry in Table 1). The results in Table 1 show that the nonlinear least squares estimavor selected predicts parameters which allow $95 \%$ oscillation damped moves and a second order method is not necessary for our conditions.

Thus, using the batch estimator to determine the necessary parameters for oscillation damped moves was successful. However, a few precautions must be taken to insure success with this method. For example, the initial guess for the initial angle must be larger than the guess for the initial angular velocity; we used nonnegative values for both. Plus, an error in the initial guesses of up to $15 \%$ of the mass and up to $20 \%$ of the length of the swinging object can be tolerated. The force sensor's coordinate frame must be aligned with gravity.

The batch estimator is based on a model which expects input data which is symmetrical about the weight of the suspended object, and cannot calculate the desired parameters accurately if the input force data is skewed. Also, the force data is expected to be reasonably continuous. Our algorithm checked to see if each force data point read was similar to the previous reading, to eliminate a large noise spike, if one occurred. If the force reading was unacceptable, another reading was taken. If a number of sampling periods passed between acceptable readings, the estimator would not converge as closely as usual (i.e., our performance function value was higher at the end of the final iteration), and the value for the period was a few percent further away from the expected value. Another effect to note is that noise in the force readings will be more significant with a lighter object, where the range of force readings is smaller, than for a heavier object. In our experiments, we used a force sensor with a range of 500 pounds and a resolution of 0.24 pounds. Thus, noise can have a larger effect with small ranges of force data. A noise nrror of just one least significant bit $(0.24 \mathrm{lb})$ is a significant percentage $(2 \%)$ of the range of our data for a typi. cal test of our lightest test objert. However, even with these potential errors the lintch, nonlincar least square estimator determinl's the model parameters which are sufficient to formulate $95 \%$ oscillation damped trajectories.

\section{Conclusions}

The batch, nonlinear least square estimator accurately predicts the parameters of a variety of simply suspended objects. The resulting oscillation damped moves using the predicted periods were successful. Thus, moving simply suspended objects with unknown parameters without in- 
ducing oscillation is possible with this method. Siveral aspects of recording the forces, including the range and continuity of the force data, and whether the data are skewed from the weight of the suspended object, can affect the operation of the estimator, resulting in less accurate results or converging to the wrong solution. Note that this method does not require a large amount of time to execute and an oscillation damped move using the estimated parameters can be done after only a few oscillations from when the force data is tiaken.

Extensions of this work should include the development and implementation of a real-time, sequential estimator to predict system parameters while the robot is continuously operating. This system architecture might include the synthesis of swing-free transportation control algorithms during manual crane operation. Our experimental equipment is currently being modified to investigate a real-time, sequential estimator.

\section{References}

[1] Jones, J. F. and Petterson, B. J., "Oscillation Damped Movernent of Suspended Objects", IEEE International Conference on Robotics and Automation, Philadelphia, PA, April, 1988.

[2] Tzes, P. A., and Yurkovish, S., "A Frequency Domain Identification Scheme for Flexible Structure Control", 27th IEEE Conference on Decision and Control, Austin, TX, December 1988.

[3] Nelson, W. L., and Mitra, D., "Load Estimation and Load-Adaptive Optimal Control for a Flexible Robot Arm", IEEE Robotics and Automation Conference, San Francisco, CA, April 1986.

[4] Jones, J. F., Petterson, B. J., and Werner, J. C., "Swing Damped Movement of Suspended Objects", SAND Report. \#87-2189, Sandia National Laboratories, to be published.
[5] Junkins, J. L., An Introduction to. Optimal Estimation of Dynamical Systems, Sijthoft' $\&$ Noordhoff International Publishers, The Netherlands, 1978 .

[6] Eisler, G. R., "FLAWLES: FLight Analysis by Weighted LEast Squares", SAND Report, Sandia National Laboratories (to be published).

[7] McDowell, J. L., "TRAP: TRajectory Analysis Program", Univerisity of Texas at Austin, Center for Advanced Study in Orbital Michanics, Austin, TX, TR77-33, 1977.

[8] Noble, B. and Daniels, J., Applied Lincar Algebra, Prentice-Hall, Englewood Cliffs, New Jersey, 1977. 

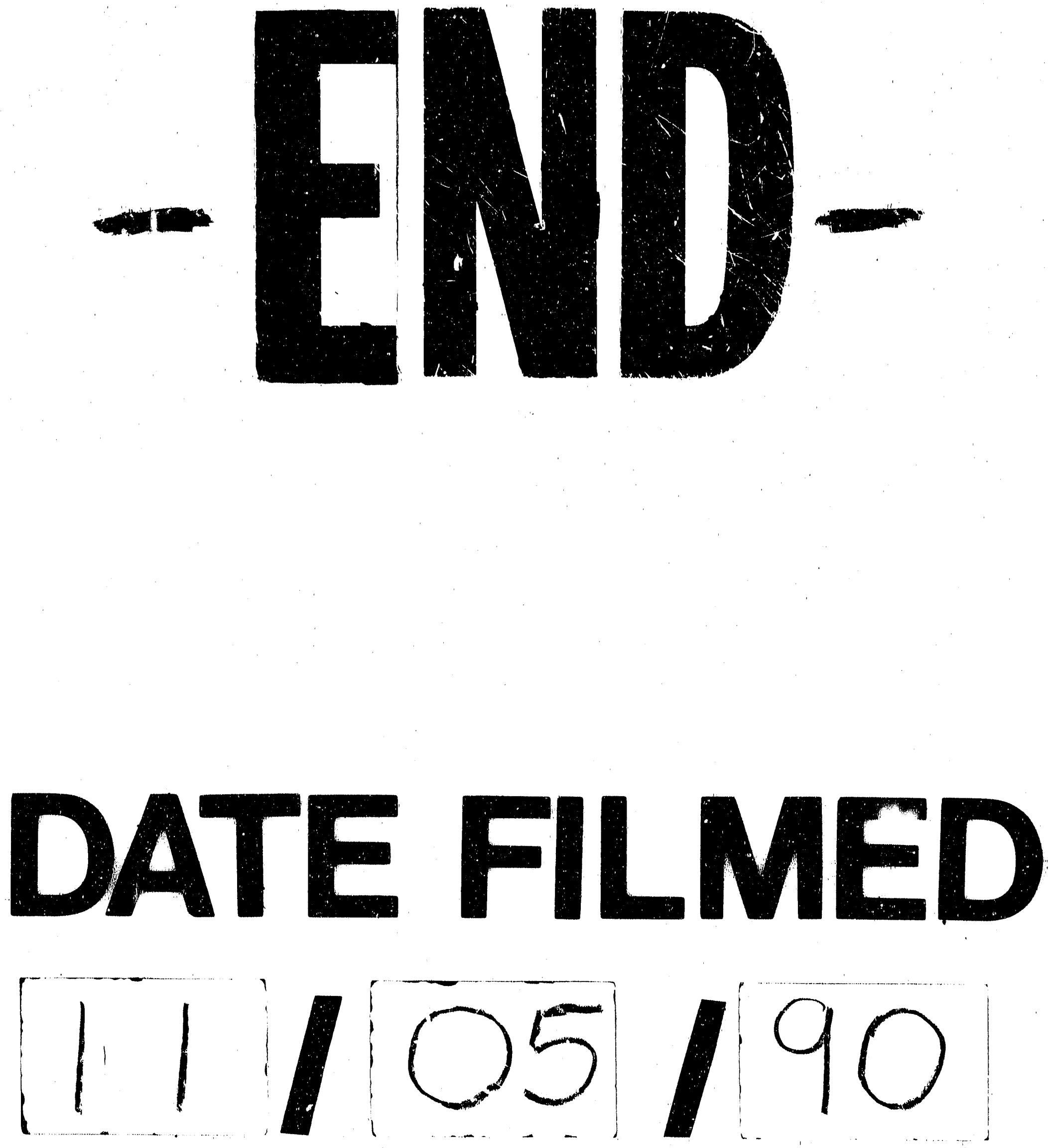
